\title{
The RS Generalized Lambda Distribution Based Calibration Model
}

\author{
Steve $\mathrm{Su}^{1}$, Abeer Hasan $^{2} \&$ Wei Ning ${ }^{2}$ \\ ${ }^{1}$ Covance Pty Ltd., Sydney, Australia; School of Mathematics and Statistics, University of Western Australia, \\ Crawley, Australia \\ ${ }^{2}$ Department of Mathematics and Statistics, Bowling Green State University, Bowling Green, USA \\ Correspondence: Steve Su, Covance Pty Ltd., Sydney, Australia; School of Mathematics and Statistics, University \\ of Western Australia, Crawley 6009, Australia. E-mail: dbarro2@gmail.com
}

Received: December 5, 2012 Accepted: January 10, 2013 Online Published: January 22, 2013

doi:10.5539/ijsp.v2n1p101 URL: http://dx.doi.org/10.5539/ijsp.v2n1p101

\begin{abstract}
We propose a flexible linear calibration model with errors from RS (Ramberg \& Schmeiser, 1974) generalized

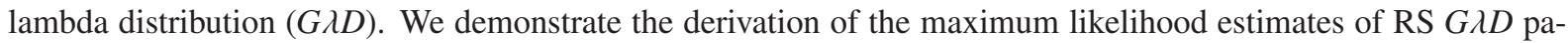
rameters and examine the estimation performance using a simulation study for sample sizes ranging from 30 to

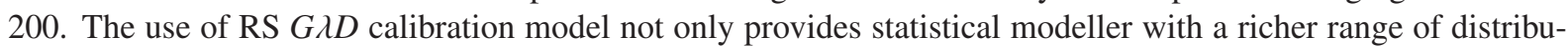
tional shapes, but can also provide more precise parameter estimates compared to the standard Normal calibration model or skewed Normal calibration model proposed by Figueiredoa, Bolfarinea, Sandovala and Limab (2010).
\end{abstract}

Keywords: generalized lambda distribution, linear calibration model, skew normal distribution, maximum likelihood estimation

\section{Introduction}

The statistical calibration model is a reverse regression technique, where we use the response variable to predict the corresponding explanatory variable. There are number of applications of this technique in science. For example, we may use radiometric dating to ascertain the age of a tree and further verify our result using tree rings. Our aim, however, is to use radiometric dating to estimate age of new trees, and the problem is whether we should minimize errors in the observation or minimize errors in age determination. There are many similar problems in substance concentration determination in biology and chemistry, physical quantities determination in physics and blood pressure/cholsterol level measurement in medicine. The literature on calibration problem has a long history, and one of the earliest works can be found in Eisenhart (1939).

The usual calibration experiment is a two stage process involving two random variables $X$ and $Y$. The first stage is known as the calibration trial, where we observe the $n$ values of the response variable $y_{1}, \cdots, y_{n}$ from a given set of explanatory values $x_{1}, \cdots, x_{n}$ and we can estimate the link function between $X$ and $Y$. The second stage is known as the calibration experiment, where we observe $k \geq 1$ value(s) of the response variable $Y$ as $y_{01}, \cdots, y_{0 k}$ which are mapped from some unknown value $x_{0}$ from the explanatory variable $X$. We can express these two stages by the following equations.

$$
\begin{gathered}
y_{i}=\alpha+\beta x_{i}+\varepsilon_{i}, \quad i=1, \cdots, n \\
y_{0 j}=\alpha+\beta x_{0}+\varepsilon_{0 j}, \quad j=1, \cdots, k,
\end{gathered}
$$

We usually assume that the errors $\varepsilon_{1}, \cdots, \varepsilon_{n}, \varepsilon_{01}, \cdots, \varepsilon_{0 k}$ are i.i.d and Normally distributed with mean 0 and variance $\sigma^{2}$. Also, $x_{1}, \cdots, x_{n}$ are known and $\alpha, \beta, x_{0}$ and $\sigma^{2}$ are unknown parameters which we need to estimate.

As an extension to Normal distribution, Azzalini (1985) introduced the skewed Normal distribution. The skewed Normal distribution is defined as

$$
g(x ; \xi, \omega, \lambda)=\frac{2}{\omega} \phi\left(\frac{x-\xi}{\omega}\right) \Phi\left(\lambda\left(\frac{x-\xi}{\omega}\right)\right),
$$

where $\phi(\cdot)$ and $\Phi(\cdot)$ are the p.d.f. and c.d.f. of a standard normal distribution respectively. Specially, when $\xi=$ 0 and $\omega=1$, we obtain the standard skewed Normal distribution. 
Based on (1.2), Figueiredoa et al. (2010) defined a skew-normal calibration model by assuming that $\varepsilon_{i}$ and $\varepsilon_{0 j}$ are i.i.d. and follow a skewed Normal distribution with $\xi=0$ denoted by $S N(0, \omega, \lambda)$. This gives us the following calibration model:

$$
\begin{aligned}
y_{i} \mid x_{i} \sim S N\left(\alpha+\beta x_{i} ; \omega ; \lambda\right), & i=1 . \cdots, n, \\
y_{0 j} \mid x_{0} \sim S N\left(\alpha+\beta x_{i} ; \omega ; \lambda\right), & j=1, \cdots, k .
\end{aligned}
$$

In (1.3), the conditional distribution of $y_{i}$ given $x_{i}$ and $y_{0 j}$ given $x_{0}$ are governed by skewed Normal distributions. This skewed Normal calibration model allows the modeller to cope with some degree of skewness in the error distribution. However, this is still limited as the skewed Normal distribution have limited range of shapes. The skewed Normal distribution still cannot handle heavy tailed, U shape, uniform, triangular or exponential upward/downward patterns. These shapes however, can be captured using $G \lambda D$ (generalized lambda distributions), and we propose a further extension to the calibration model by using RS $G \lambda D$.

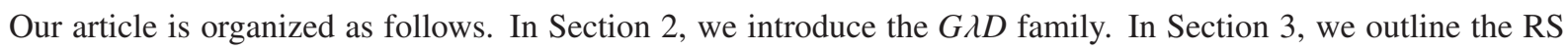
$G \lambda D$ calibration model and discuss possible ways to estimate parameters of the model using maximum likelihood estimation. In Section 4, we demonstrate the estimation performance of our proposed model across a range of different sample sizes from 30 to 200. As a further test to our proposed model to the literature, we compare the performance of RS G $\lambda D$ calibration model against Normal and skewed Normal calibration model with respect to a real life dataset used by Figueiredoa et al. (2010) in Section 5. A discussion of our proposed method is given in Section 6.

\section{Generalized Lambda Distributions}

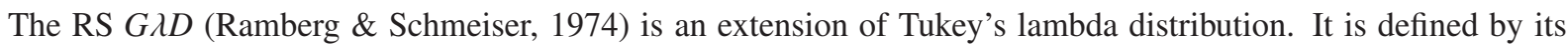
inverse distribution function:

$$
F^{-1}(u)=\lambda_{1}+\frac{u^{\lambda_{3}}-(1-u)^{\lambda_{4}}}{\lambda_{2}} \quad 0 \leq u \leq 1
$$

From (2.1), $\lambda_{1}, \lambda_{2}, \lambda_{3}, \lambda_{4}$ are respectively the location, inverse scale and shape 1 and shape 2 parameters. Karian and Dudewicz (2000) noted that $G \lambda D$ is defined only if $\frac{\lambda_{2}}{\lambda_{3} u^{\lambda_{3}-1}+\lambda_{4}(1-u)^{\lambda_{4}-1}} \geq 0$ for $0 \leq u \leq 1$. The conditions

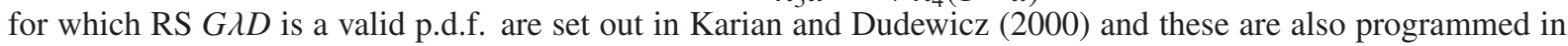
GLDEX package in $\mathrm{R}$ (Su, 2010, 2007a).

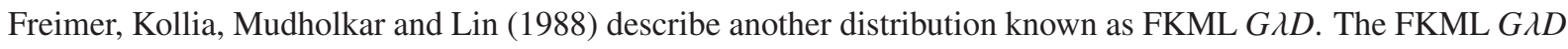
can be written as:

$$
F^{-1}(u)=\lambda_{1}+\frac{\frac{u^{\lambda_{3}}-1}{\lambda_{3}}-\frac{(1-u)^{\lambda_{4}}-1}{\lambda_{4}}}{\lambda_{2}} \quad 0 \leq u \leq 1
$$

Under (2.2), $\lambda_{1}, \lambda_{2}, \lambda_{3}, \lambda_{4}$ are respectively the location, inverse scale and shape 1 and shape 2 parameters.

The fundamental motivation for the development of FKML G $\lambda D$ is that the distribution is defined over all $\lambda_{3}$ and $\lambda_{4}$ (Freimer et al., 1988). The only restriction on FKML $G \lambda D$ is $\lambda_{2}>0$. This is more convenient to deal with computationally than RS $G \lambda D$ and hence it is sometimes the preferred $G \lambda D$ for some researchers.

We restrict our attention in this article to the more difficult problem of fitting RS G $\lambda D$ calibration model to data. Without loss of generality, the method we outlined below can be easily adapted to build FKML G $\lambda D$ calibration model.

\section{Statistical Model}

\subsection{GגD Based Calibration Model}

We consider the following usual calibration model:

$$
\begin{gathered}
y_{i}=\alpha+\beta x_{i}+\epsilon_{i}, \quad i=1, \cdots, n, \\
y_{0 j}=\alpha+\beta x_{0}+\epsilon_{j}, \quad j=1, \cdots, k .
\end{gathered}
$$

We assume that $\epsilon_{i}$ and $\epsilon_{j}$ are i.i.d. $G \lambda D\left(0, \lambda_{2}, \lambda_{3}, \lambda_{4}\right)$. In general, we consider $x_{1}, \cdots, x_{n}$ to be known and fixed and $\alpha, \beta, \lambda_{2}, \lambda_{3}$ and $\lambda_{4}$ are parameters we need to estimate. Our $G \lambda D$ calibration model takes the following form:

$$
y_{i} \mid x_{i} \sim G \lambda D\left(\alpha+\beta x_{i}, \lambda_{2}, \lambda_{3}, \lambda_{4}\right)
$$




$$
y_{0 j} \mid x_{0} \sim G \lambda D\left(\alpha+\beta x_{0}, \lambda_{2}, \lambda_{3}, \lambda_{4}\right) .
$$

Consequently, the likelihood function for RS $G \lambda D$ is:

$$
L\left(\boldsymbol{\theta}, \boldsymbol{y}, \boldsymbol{y}_{0}\right)=\prod_{i=1}^{n} \frac{\lambda_{2}}{\lambda_{3} z_{i}^{\lambda_{3}-1}+\lambda_{4}\left(1-z_{i}\right)^{\lambda_{4}-1}} \cdot \prod_{j=1}^{k} \frac{\lambda_{2}}{\lambda_{3} z_{j}^{\lambda_{3}-1}+\lambda_{4}\left(1-z_{j}\right)^{\lambda_{4}-1}},
$$

where

$$
\begin{gathered}
y_{i}=\left(\alpha+\beta x_{i}\right)+\frac{z_{i}^{\lambda_{3}}-\left(1-z_{i}\right)^{\lambda_{4}}}{\lambda_{2}}, \\
y_{0 j}=\left(\alpha+\beta x_{0}\right)+\frac{z_{j}^{\lambda_{3}}-\left(1-z_{j}\right)^{\lambda_{4}}}{\lambda_{2}},
\end{gathered}
$$

and $0 \leq z_{i}, z_{j} \leq 1, \boldsymbol{\theta}=\left(\alpha, \beta, x_{0}, \lambda_{2}, \lambda_{3}, \lambda_{4}\right)$.

\subsection{Estimation of Parameters}

From (3.5), we obtain the following log likelihood function:

$$
\log L\left(\boldsymbol{\theta}, \boldsymbol{y}, \boldsymbol{y}_{\mathbf{0}}\right)=\sum_{i=1}^{n} \log \left(f_{1}\left(\boldsymbol{\theta}, y_{i}\right)\right)+\sum_{j=1}^{k} \log \left(f_{2}\left(\boldsymbol{\theta}, y_{0 j}\right)\right)
$$

where

$$
\begin{aligned}
& f_{1}\left(\boldsymbol{\theta}, y_{i}\right)=\frac{\lambda_{2}}{\lambda_{3} z_{i}^{\lambda_{3}-1}+\lambda_{4}\left(1-z_{i}\right)^{\lambda_{4}-1}}, \\
& f_{2}\left(\boldsymbol{\theta}, y_{0 j}\right)=\frac{\lambda_{2}}{\lambda_{3} z_{j}^{\lambda_{3}-1}+\lambda_{4}\left(1-z_{j}\right)^{\lambda_{4}-1}}
\end{aligned}
$$

Taking the derivative of (3.6), we obtain the following:

$$
\frac{\partial \log L(\boldsymbol{\theta})}{\partial \boldsymbol{\theta}}=\sum_{i=1}^{n} \frac{1}{f_{1}} \frac{\partial f_{1}}{\partial \boldsymbol{\theta}}+\sum_{j=1}^{k} \frac{1}{f_{2}} \frac{\partial f_{2}}{\partial \boldsymbol{\theta}},
$$

where $\boldsymbol{\theta}=\left(\alpha, \beta, x_{0}, \lambda_{2}, \lambda_{3}, \lambda_{4}\right)$.

Theoretically, the MLE of $\theta$ is the solution of (3.7) when it is set to be equal to 0 . The derivatives $\frac{\partial f_{1}}{\partial \theta}$ and $\frac{\partial f_{2}}{\partial \theta}$ are given below.

$$
\begin{aligned}
\frac{\partial f_{1}}{\partial \lambda_{2}} & =\frac{\partial f_{1}}{\partial z_{i}} \cdot \frac{\partial z_{i}}{\partial y_{i}} \cdot \frac{\partial y_{i}}{\partial \lambda_{2}} \\
& =\left(\lambda_{2} \frac{-\lambda_{3}\left(\lambda_{3}-1\right) z_{i}^{\lambda_{3}-2}+\lambda_{4}\left(\lambda_{4}-1\right)\left(1-z_{i}\right)^{\lambda_{4}-2}}{\left(\lambda_{3} z_{i}^{\lambda_{3}-1}+\lambda_{4}\left(1-z_{i}\right)^{\lambda_{4}-1}\right)^{2}}\right) \cdot\left(\frac{\lambda_{2}}{\lambda_{3} z_{i}^{\lambda_{3}-1}+\lambda_{4}\left(1-z_{i}\right)^{\lambda_{4}-1}}\right) \cdot\left(-\frac{z_{i}^{\lambda_{3}}-\left(1-z_{i}\right)^{\lambda_{4}}}{\lambda_{2}^{2}}\right) \\
& =\frac{\left[\lambda_{3}\left(\lambda_{3}-1\right) z_{i}^{\lambda_{3}-2}-\lambda_{4}\left(\lambda_{4}-1\right)\left(1-z_{i}\right)^{\lambda_{4}-2}\right]\left(z_{i}^{\lambda_{3}}-\left(1-z_{i}\right)^{\lambda_{4}}\right)}{\left(\lambda_{3} z_{i}^{\lambda_{3}-1}+\lambda_{4}\left(1-z_{i}\right)^{\lambda_{4}-1}\right)^{3}} \\
\frac{\partial f_{1}}{\partial \lambda_{3}} & =\left(-\lambda_{2}\right) \frac{\left[\lambda_{3}\left(\lambda_{3}-1\right) z_{i}^{\lambda_{3}-2}-\lambda_{4}\left(\lambda_{4}-1\right)\left(1-z_{i}\right)^{\lambda_{4}-2}\right]\left(z_{i}^{\lambda_{3}} \log z_{i}\right)}{\left(\lambda_{3} z_{i}^{\lambda_{3}-1}+\lambda_{4}\left(1-z_{i}\right)^{\lambda_{4}-1}\right)^{3}} \\
\frac{\partial f_{1}}{\partial \lambda_{4}} & =\lambda_{2} \frac{\left[\lambda_{3}\left(\lambda_{3}-1\right) z_{i}^{\lambda_{3}-2}-\lambda_{4}\left(\lambda_{4}-1\right)\left(1-z_{i}\right)^{\lambda_{4}-2}\right]\left(\left(1-z_{i}\right)^{\lambda_{3}} \log \left(1-z_{i}\right)\right)}{\left(\lambda_{3} z_{i}^{\lambda_{3}-1}+\lambda_{4}\left(1-z_{i}\right)^{\lambda_{4}-1}\right)^{3}} \\
\frac{\partial f_{1}}{\partial \alpha} & =\left(-\lambda_{2}^{2}\right) \frac{\left[\lambda_{3}\left(\lambda_{3}-1\right) z_{i}^{\lambda_{3}-2}-\lambda_{4}\left(\lambda_{4}-1\right)\left(1-z_{i}\right)^{\lambda_{4}-2}\right]}{\left(\lambda_{3} z_{i}^{\lambda_{3}-1}+\lambda_{4}\left(1-z_{i}\right)^{\lambda_{4}-1}\right)^{3}} \\
\frac{\partial f_{1}}{\partial \beta} & =\left(-\lambda_{2}^{2}\right) \frac{\left[\lambda_{3}\left(\lambda_{3}-1\right) z_{i}^{\lambda_{3}-2}-\lambda_{4}\left(\lambda_{4}-1\right)\left(1-z_{i}\right)^{\lambda_{4}-2}\right] \cdot x_{i}}{\left(\lambda_{3} z_{i}^{\lambda_{3}-1}+\lambda_{4}\left(1-z_{i}\right)^{\lambda_{4}-1}\right)^{3}} \\
\frac{\partial f_{2}}{\partial \lambda_{2}} & =\frac{\left[\lambda_{3}\left(\lambda_{3}-1\right) z_{j}^{\lambda_{3}-2}-\lambda_{4}\left(\lambda_{4}-1\right)\left(1-z_{j}\right)^{\lambda_{4}-2}\right]\left(z_{j}^{\lambda_{3}}-\left(1-z_{j}\right)^{\lambda_{4}}\right)}{\left(\lambda_{3} z_{i}^{\lambda_{3}-1}+\lambda_{4}\left(1-z_{j}\right)^{\lambda_{4}-1}\right)^{3}}
\end{aligned}
$$




$$
\begin{aligned}
& \frac{\partial f_{2}}{\partial \lambda_{3}}=\left(-\lambda_{2}\right) \frac{\left[\lambda_{3}\left(\lambda_{3}-1\right) z_{j}^{\lambda_{3}-2}-\lambda_{4}\left(\lambda_{4}-1\right)\left(1-z_{j}\right)^{\lambda_{4}-2}\right]\left(z_{j}^{\lambda_{3}} \log z_{j}\right)}{\left(\lambda_{3} z_{i}^{\lambda_{3}-1}+\lambda_{4}\left(1-z_{i}\right)^{\lambda_{4}-1}\right)^{3}} \\
& \frac{\partial f_{1}}{\partial \lambda_{4}}=\lambda_{2} \frac{\left[\lambda_{3}\left(\lambda_{3}-1\right) z_{j}^{\lambda_{3}-2}-\lambda_{4}\left(\lambda_{4}-1\right)\left(1-z_{j}\right)^{\lambda_{4}-2}\right]\left(\left(1-z_{j}\right)^{\lambda_{3}} \log \left(1-z_{j}\right)\right)}{\left(\lambda_{3} z_{j}^{\lambda_{3}-1}+\lambda_{4}\left(1-z_{j}\right)^{\lambda_{4}-1}\right)^{3}} \\
& \frac{\partial f_{2}}{\partial \alpha}=\left(-\lambda_{2}^{2}\right) \frac{\left[\lambda_{3}\left(\lambda_{3}-1\right) z_{j}^{\lambda_{3}-2}-\lambda_{4}\left(\lambda_{4}-1\right)\left(1-z_{j}\right)^{\lambda_{4}-2}\right]}{\left(\lambda_{3} z_{j}^{\lambda_{3}-1}+\lambda_{4}\left(1-z_{j}\right)^{\lambda_{4}-1}\right)^{3}} \\
& \frac{\partial f_{2}}{\partial \beta}=\left(-\lambda_{2}^{2}\right) \frac{\left[\lambda_{3}\left(\lambda_{3}-1\right) z_{j}^{\lambda_{3}-2}-\lambda_{4}\left(\lambda_{4}-1\right)\left(1-z_{j}\right)^{\lambda_{4}-2}\right] \cdot x_{0}}{\left(\lambda_{3} z_{j}^{\lambda_{3}-1}+\lambda_{4}\left(1-z_{j}\right)^{\lambda_{4}-1}\right)^{3}} \\
& \frac{\partial f_{2}}{\partial x_{0}}=\left(-\lambda_{2}^{2}\right) \frac{\left[\lambda_{3}\left(\lambda_{3}-1\right) z_{j}^{\lambda_{3}-2}-\lambda_{4}\left(\lambda_{4}-1\right)\left(1-z_{j}\right)^{\lambda_{4}-2}\right] \cdot \beta}{\left(\lambda_{3} z_{j}^{\lambda_{3}-1}+\lambda_{4}\left(1-z_{j}\right)^{\lambda_{4}-1}\right)^{3}}
\end{aligned}
$$

It is difficult to obtain the exact solutions of setting (3.7) to zero using the above formulations, owing to the fact

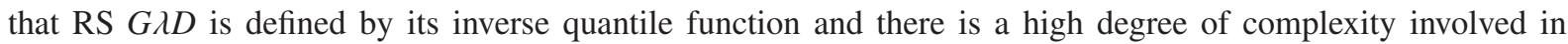
solving the above equations. As an alternative, we carry out the maximum likelihood estimation by maximising (3.6) directly using Nelder-Mead optimisation algorithm as is customary done for maximum likelihood estimation problems involving $G \lambda D$ (see Su, 2010, 2007a, 2007b). This is a preferred and more reliable method of estimation as opposed to trying to satisfy the exact conditions to which all of the above equations equal to zero. The GLDEX package in $\mathrm{R}(\mathrm{Su}, 2010,2007 \mathrm{a})$ facilitates the Nelder-Mead optimisation algorithm for $G \lambda D$.

Our algorithm is as follows:

1) Generate a set of initial values for $\alpha, \beta, x_{0}, \lambda_{2}, \lambda_{3}, \lambda_{4}$. There are a number of strategies that can be used to determine the best set of initial values. One strategy is to generate initial values $\alpha, \beta, x_{0}$ using Normal or skewed Normal calibration model and then generate some low discrepancy quasi random numbers for $\lambda_{2}, \lambda_{3}, \lambda_{4}$ over a range of values and select the set of initial values that maximises (3.6). Alternatively all initial values can be randomly generated using low discrepancy quasi random numbers.

2) Set $\lambda_{1}=\alpha+\beta x_{0}$.

3) Check that $\operatorname{G\lambda D}\left(\lambda_{1}, \lambda_{2}, \lambda_{3}, \lambda_{4}\right)$ is a valid statistical distribution, this can be done using GLDEX package in R.

4) Check the minimal support of $G \lambda D\left(\lambda_{1}, \lambda_{2}, \lambda_{3}, \lambda_{4}\right)$ is lower or equal to the lowest value of $y_{0}$. Similarly, check that the maximum support of $G \lambda D\left(\lambda_{1}, \lambda_{2}, \lambda_{3}, \lambda_{4}\right)$ is greater or equal to the largest value of $y_{0}$. This is to ensure that the fitted $G \lambda D$ will span the entire dataset. If these conditons are not met, choose another set of initial values and repeat from 2).

5) Conduct Nelder Mead optimisation by maximising (3.6) directly using the above initial values to obtain the required estimates.

\section{Simulations}

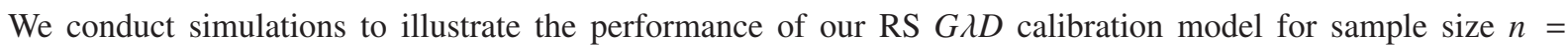
$30,50,100$ and 200 with $\alpha=3, \beta=1.5, x_{0}=15$ or $40, \lambda_{3}=10, \lambda_{4}=1$, and $\lambda_{2}=2,5,10$. We further generate $x_{1}, x_{2}, \cdots, x_{n}$ from Uniform $(10,30)$, and we set $k=1$. We use the true parameters as our initial values to kick start the optimisation process to obtain our MLE estimate for $x_{0}$.

We repeat this process 1000 times, which give us $1000 \hat{x}_{0 m}$ estimates of $x_{0}$. The mean $\hat{x}_{0}$, $\operatorname{Bias}\left(x_{0}\right)$ and $\operatorname{MSE}\left(x_{0}\right)$ are calculated as follows:

$$
\begin{aligned}
\bar{x}_{0} & =\frac{1}{1000} \sum_{m=1}^{1000} \hat{x}_{0 m} \\
\operatorname{Bias}\left(x_{0}\right) & =\frac{1}{1000} \sum_{m=1}^{1000}\left(\hat{x}_{0 m}-x_{0}\right) \\
\operatorname{MSE}\left(x_{0}\right) & =\frac{1}{1000} \sum_{m=1}^{1000}\left(\hat{x}_{0 m}-x_{0}\right)^{2}
\end{aligned}
$$


The results of above simulations are shown in Tables 1 and 2. As expected, the MSE decreases as we increase the sample size or increase the value of inverse scale parameter $\lambda_{2}$. In terms of bias, we observe that the performance appear to be fairly consistent across sample sizes, this gives confidence in the use of RS G $\lambda D$ calibration model for smaller samples, even though there are are more parameters that need to be estimated from this model. There also appears to be a tendency for RS G $\lambda D$ calibration model to slightly overestimate as nearly all the bias results are positive. Increasing the shape parameter $\lambda_{3}$ does not always result in increase in MSE, this is because the shape parameter spaces of $\lambda_{3}$ and $\lambda_{4}$ for RS G $\lambda D$ are fairly complex.

Table 1. Simulations results with $x_{0}=15, \alpha=3, \beta=1.5, \lambda_{4}=1$

\begin{tabular}{|c|c|c|c|c|c|c|c|c|c|c|}
\hline \multirow[b]{2}{*}{$n$} & \multirow[b]{2}{*}{$\lambda_{3}$} & \multicolumn{3}{|c|}{$\lambda_{2}=2$} & \multicolumn{3}{|c|}{$\lambda_{2}=5$} & \multicolumn{3}{|c|}{$\lambda_{2}=10$} \\
\hline & & $\hat{x}_{0}$ & Bias & MSE & $\hat{x}_{0}$ & Bias & MSE & $\hat{x}_{0}$ & Bias & MSE \\
\hline 30 & 10 & 15.1105 & 0.1105 & 0.0263 & 15.0386 & 0.0386 & 0.0042 & 15.0178 & 0.0178 & 0.0010 \\
\hline 50 & 10 & 15.0944 & 0.0944 & 0.0232 & 15.0352 & 0.0352 & 0.0040 & 15.0172 & 0.0172 & 0.0010 \\
\hline 100 & 10 & 15.0994 & 0.0994 & 0.0184 & 15.0396 & 0.0396 & 0.0035 & 15.0185 & 0.0185 & 0.0008 \\
\hline 200 & 10 & 15.1053 & 0.1053 & 0.0166 & 15.0340 & 0.0340 & 0.0030 & 15.0173 & 0.0173 & 0.0007 \\
\hline 30 & 5 & 15.1430 & 0.1430 & 0.0292 & 15.0578 & 0.0578 & 0.0056 & 15.0285 & 0.0285 & 0.0012 \\
\hline 50 & 5 & 15.1445 & 0.1445 & 0.0270 & 15.0530 & 0.0530 & 0.0047 & 15.0292 & 0.0292 & 0.0012 \\
\hline 100 & 5 & 15.1381 & 0.1381 & 0.0214 & 15.0531 & 0.0531 & 0.0043 & 15.0264 & 0.0264 & 0.0010 \\
\hline 200 & 5 & 15.1429 & 0.1429 & 0.0187 & 15.0534 & 0.0534 & 0.0038 & 15.0227 & 0.0227 & 0.0009 \\
\hline 30 & 1 & 15.0271 & 0.0271 & 0.0244 & 15.0014 & 0.0014 & 0.0061 & 15.0040 & 0.0040 & 0.0017 \\
\hline 50 & 1 & 15.0367 & 0.0367 & 0.0169 & 15.0030 & 0.0030 & 0.0048 & 14.9993 & -0.0007 & 0.0014 \\
\hline 100 & 1 & 15.0292 & 0.0292 & 0.0084 & 15.0093 & 0.0093 & 0.0030 & 15.0029 & 0.0029 & 0.0010 \\
\hline 200 & 1 & 15.0262 & 0.0262 & 0.0052 & 15.0130 & 0.0130 & 0.0016 & 15.0022 & 0.0022 & 0.0007 \\
\hline
\end{tabular}

Table 2. Simulations results with $x_{0}=40, \alpha=3, \beta=1.5, \lambda_{4}=1$

\begin{tabular}{|c|c|c|c|c|c|c|c|c|c|c|}
\hline \multirow[b]{2}{*}{$n$} & \multirow[b]{2}{*}{$\lambda_{3}$} & \multicolumn{3}{|c|}{$\lambda_{2}=2$} & \multicolumn{3}{|c|}{$\lambda_{2}=5$} & \multicolumn{3}{|c|}{$\lambda_{2}=10$} \\
\hline & & $\hat{x}_{0}$ & Bias & MSE & $\hat{x}_{0}$ & Bias & MSE & $\hat{x}_{0}$ & Bias & MSE \\
\hline 30 & 10 & 40.1070 & 0.1070 & 0.0259 & 40.0375 & 0.0375 & 0.0049 & 40.0189 & 0.0189 & 0.0012 \\
\hline 50 & 10 & 40.1051 & 0.1051 & 0.0235 & 40.0388 & 0.0388 & 0.0039 & 40.0177 & 0.0177 & 0.0009 \\
\hline 100 & 10 & 40.1077 & 0.1077 & 0.0205 & 40.0353 & 0.0353 & 0.0031 & 40.0188 & 0.0188 & 0.0008 \\
\hline 200 & 10 & 40.1088 & 0.1088 & 0.0169 & 40.0387 & 0.0387 & 0.0028 & 40.0184 & 0.0184 & 0.0008 \\
\hline 30 & 5 & 40.1339 & 0.1339 & 0.0319 & 40.0557 & 0.0557 & 0.0064 & 40.0288 & 0.0288 & 0.0014 \\
\hline 50 & 5 & 40.1391 & 0.1391 & 0.0302 & 40.0554 & 0.0554 & 0.0046 & 40.0280 & 0.0280 & 0.0013 \\
\hline 100 & 5 & 40.1405 & 0.1405 & 0.0232 & 40.0479 & 0.0479 & 0.0039 & 40.0264 & 0.0264 & 0.0010 \\
\hline 200 & 5 & 40.1538 & 0.1538 & 0.0236 & 40.0474 & 0.0474 & 0.0035 & 40.0205 & 0.0205 & 0.0007 \\
\hline 30 & 1 & 40.0331 & 0.0331 & 0.0290 & 39.9984 & -0.0016 & 0.0058 & 40.0035 & 0.0035 & 0.0016 \\
\hline 50 & 1 & 40.0348 & 0.0348 & 0.0159 & 40.0031 & 0.0031 & 0.0045 & 40.0022 & 0.0022 & 0.0013 \\
\hline 100 & 1 & 40.0311 & 0.0311 & 0.0099 & 40.0078 & 0.0078 & 0.0024 & 39.9996 & -0.0004 & 0.0009 \\
\hline 200 & 1 & 40.0217 & 0.0217 & 0.0036 & 40.0114 & 0.0114 & 0.0017 & 40.0031 & 0.0031 & 0.0007 \\
\hline
\end{tabular}

Table 3. Simulations results with $x_{0}=15, \alpha=3, \beta=1.5$, true error distribution $\operatorname{GEV}(0.1860,0.4016,0.1511)$ is approximated by $\operatorname{RS} G \lambda D$ with $\lambda_{1}=0, \lambda_{2} \approx-0.0374, \lambda_{3} \approx-0.0027, \lambda_{4} \approx-0.0212$

\begin{tabular}{rccc}
\hline$n$ & $\hat{x}_{0}$ & Bias & MSE \\
\hline 30 & 15.3140 & 0.3140 & 0.2149 \\
50 & 15.3269 & 0.3269 & 0.2154 \\
100 & 15.2815 & 0.2815 & 0.1774 \\
200 & 15.2860 & 0.2860 & 0.1689 \\
\hline
\end{tabular}

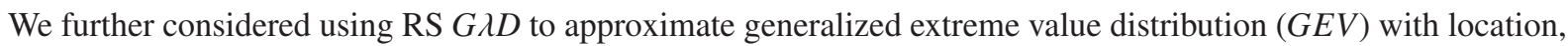
scale and shape parameters being $0.1860,0.4016,0.1511$ respectively. We choose $\operatorname{RS} G \lambda D$ with $\lambda_{1}=0, \lambda_{2} \approx$ $-0.0374, \lambda_{3} \approx-0.0027, \lambda_{4} \approx-0.0212$ for this demonstration (Figure 1 ). We then generate simulated data based

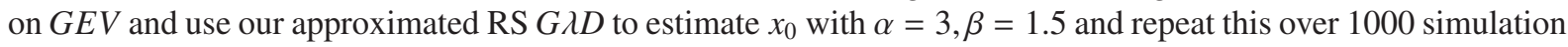
runs. The result of this simulation is given in Table 3. We observe that the RS G $\lambda D$ calibration model tends to overestimate the true $x_{0}$ by a small margin, but the bias appears to decrease as sample size increases. 


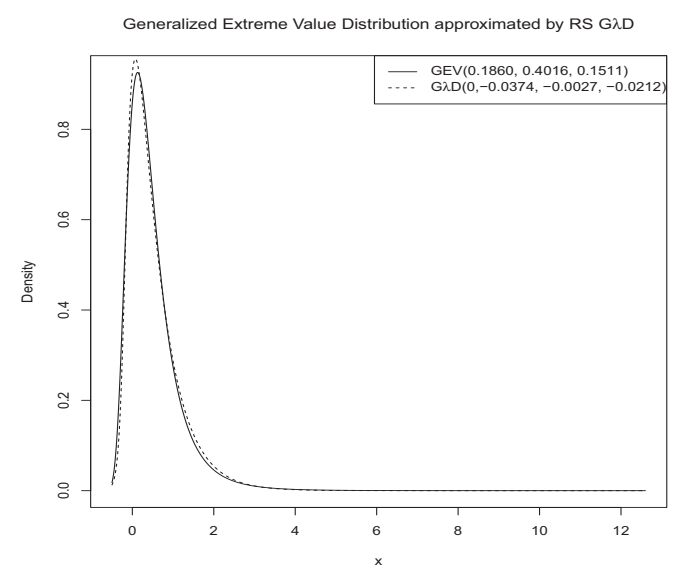

Figure 1. Approximating $G E V$ using RS $G \lambda D$

\section{Application}

We apply the RS $G \lambda D$ calibration model to a dataset which measures teenager testicular volume $\left(\mathrm{ml}^{3}\right)$. This dataset is from Chipkevitch, Nishimura, Tu and Galea-Rajas (1996) and consists of 42 observations. Figueiredoa et al. (2010) considered two measurement methods from Chipkevitch et al. (1996): dimensional measurement with a caliper (DM) and measurement by ultrasonography (US) and the data is given in Table 4. In their paper, Figueiredoa et al. (2010) consider the $x_{0}$ value of 16.4 , which is observed twice by ultrasonography. They subsequently treated this value as unknown, with corresponding $y_{0 j}$ values of $y_{01}=10.3$ and $y_{02}=17.3$. Then, they estimate $x_{0}$ using their skewed Normal calibration model and compared this with the standard Normal calibration model. We did the

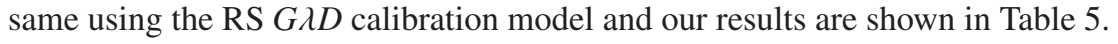

Table 4. Measurements obtained by dimensional measurement with a caliper (DM) and by ultrasonography (US) from the right testis for 42 teenagers, in $m l^{3}$

\begin{tabular}{|c|c|c|c|c|c|c|c|c|c|c|c|}
\hline $\mathrm{DM}$ & US & $\mathrm{DM}$ & US & $\mathrm{DM}$ & US & $\mathrm{DM}$ & US & $\mathrm{DM}$ & US & $\mathrm{DM}$ & US \\
\hline 5.9 & 5 & 17.3 & 16.4 & 7.2 & 6.7 & 4.8 & 5.7 & 17.3 & 17.6 & 5.9 & 5.3 \\
\hline 6.8 & 7.4 & 7.9 & 10 & 16.3 & 20 & 3.1 & 2.6 & 4.4 & 4.1 & 16.3 & 18.8 \\
\hline 5 & 5.7 & 11.4 & 12.7 & 12.2 & 13.9 & 4.4 & 6.1 & 4.1 & 2.7 & 10.3 & 9.4 \\
\hline 6 & 6.2 & 11.1 & 10.2 & 10.8 & 9.1 & 8.8 & 10.4 & 15.3 & 16.5 & 13 & 14.1 \\
\hline 7.9 & 9.1 & 3.9 & 4.5 & 8.4 & 9.3 & 13 & 14.8 & 4.5 & 5.6 & 22.1 & 20.9 \\
\hline 10.3 & 16.4 & 9.7 & 11 & 10.6 & 11.5 & 8.2 & 9.6 & 11.3 & 9.2 & 9.7 & 9.7 \\
\hline 19.8 & 15.7 & 8.8 & 8.5 & 11.6 & 13.7 & 2 & 3 & 6.1 & 5.4 & 8.1 & 8.9 \\
\hline
\end{tabular}

Table 5. A comparison of linear calibration models

\begin{tabular}{|c|c|c|c|c|c|c|}
\hline \multirow[b]{2}{*}{ Parameter } & \multicolumn{2}{|c|}{ RS $G \lambda D$ model } & \multicolumn{2}{|c|}{$S N$ model } & \multicolumn{2}{|c|}{ Normal model } \\
\hline & Estimate & Stdev. & Estimate & Stdev. & Estimate & Stdev. \\
\hline$\alpha$ & 0.014 & 0.497 & -0.69 & - & 0.32 & 0.56 \\
\hline$\beta$ & 0.855 & 0.035 & 0.86 & 0.07 & 0.92 & 0.05 \\
\hline$\sigma$ & - & - & 2.13 & - & 1.55 & 0.17 \\
\hline$x_{0}$ & 12.128 & 0.963 & 12.66 & 1.81 & 14.58 & 1.24 \\
\hline$\lambda$ & - & - & 2.16 & 1.73 & - & - \\
\hline$\lambda_{2}$ & 0.146 & 0.355 & - & - & - & - \\
\hline$\lambda_{3}$ & -0.030 & 0.061 & - & - & - & - \\
\hline$\lambda_{4}$ & -0.162 & 0.184 & - & - & - & - \\
\hline AIC & 150.36 & & 160.69 & & 163.74 & \\
\hline BIC & 160.79 & & 169.38 & & 170.69 & \\
\hline HQ & 144.58 & & 156.55 & & 161.15 & \\
\hline
\end{tabular}

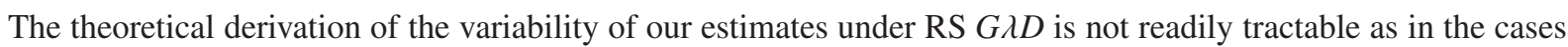
of skewed Normal and Normal distributions. As we need to numerically derive our calculations, small errors in 
numerical procedures could accumulate into large errors even if we could evaluate the exact theoretical solution. As a workaround, we adopt the following procedure. Once we obtained the parameters of our model, $\alpha, \beta, x_{0}, \lambda_{2}$, $\lambda_{3}, \lambda_{4}$, we conduct simulations to estimate the variability of our estimate. We use our estimated parameters from the RS G $\lambda D$ calibration model and $x_{i}$ (excluding $x_{i}=16.4$ ) from the original data to randomly generate $y_{0 j}$ and $y_{i}$ according to (3.1) and (3.2). We then maximise the likelihood in (3.6) using Nelder Mead Simplex algorithm with initial values being our original estimated parameters. We repeat the process 1000 times and calculate the sample standard deviations of our estimated parameters.

Table 5 lists the estimated parameters and their standard deviations from RS G $\lambda D$, skewed Normal and Normal calibration models. We compute the Akaike, Bayesian and Hannan-Quinn information criterion (AIC, BIC, and HQ) to allow model selection between three models. All three criterion favors the RS $G \lambda D$ calibration model. In

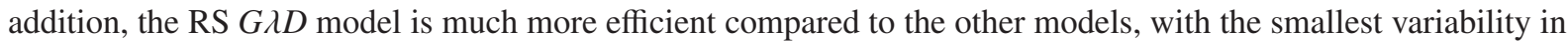
its parameter estimates.

\section{Concluding Remarks}

We propose a new calibration model with RS G $\lambda D$ errors, which is an extremely flexible model that can cope with a wide range of different error distributions. Our method also lends to the development of FKML G $\lambda D$ calibration model, which may have better properties with regard to numerical convergence. Our simulations studies suggest our proposed model perform well for small sample sizes across a range of inverse scale and shape parameters of RS $G \lambda D$. We further demonstrate that the RS G $\lambda D$ calibration model can outperform skewed Normal or Normal calibration model, with lower AIC, BIC and HQ information criterion and lower variability in our parameter estimates in the context of a real life data. These simulation results are promising and future statistical models should aim to develop statistical technique that are tailored to data, rather than requiring empirical data to satisfy a particular statistical model. One possible extension of our model is the development of a mixture RS G $\lambda D$ calibration model, which would extend the flexibility of our model even further but also present a very challenging problem for data with small samples.

\section{References}

Azzalini, A. (1985). A class of distributions which includes the normal one. Scandinavian Journal of Statistics, $12,171-178$.

Chipkevitch, E., Nishimura, R., Tu, D., \& Galea-Rajas, M. (1996). Clinical measurements of testicular volume in adolescents: Comparison of the reliability of 5 methods. The Journal of Urology, 156, 2050-2053. http://dx.doi.org/10.1016/S0022-5347(01)65433-8

Eisenhart, C. (1939). The interpretation of certain regression methods and their use in biological and industrial research. Annals of Mathematical Statistics, 10, 162-186. http://dx.doi.org/10.1214/aoms/1177732214

Figueiredoa, C., Bolfarinea, H., Sandovala, M., \& Limab, C. (2010). On the skew-normal calibration model. Journal of Applied Statistics, 37(3), 435-451. http://dx.doi.org/10.1080/02664760802715906

Freimer, M., Kollia, G., Mudholkar, G. S., \& Lin, C. T. (1988). A study of the generalised tukey lambda family. Communications in Statistics-Theory and Methods, 3547-3567. http://dx.doi.org/10.1080/03610928808829820

Karian, Z. A., \& Dudewicz, E. J. (2000). Fitting statistical distributions: The generalized lambda distribution and generalised bootstrap methods. New York: Chapman and Hall. http://dx.doi.org/10.1201/9781420038040

Ramberg, J. S., \& Schmeiser, B. W. (1974). An approximate method for generating asymmetric random variables. Communications of the Association for Computing Machinery, 17, 78-82. http://dx.doi.org/10.1145/360827.360840

Su, S. (2007a). Fitting single and mixture of generalised lambda distributions to data via discretized and maximum likelihood methods: GLDEX in R. Journal of Statistical Software, 21(9).

$\mathrm{Su}$, S. (2007b). Numerical maximum log likelihood estimation for generalized lambda distributions. Computational Statistics and Data Analysis, 51(8), 3983-3998. http://dx.doi.org/10.1016/j.csda.2006.06.008

Su, S. (2010). Handbook of distribution fitting methods with R. In E. Karian, \& Z. Dudewicz (Eds.), Fitting GLD to data Using the GLDEX 1.0.4 in R (Chap. 15). CRC Press. 